\title{
Mucosubstances in neoplasms of the human colon and rectum
}

\author{
S. G. SUBBUSWAMY1
}

From the Department of Pathology, Newcastle General Hospital, Newcastle

SUMMARY The patterns of mucosubstances secreted in the normal human colon and rectum, in benign tumours, and in and around malignant tumours of the colon and rectum have been studied.

Very little neutral mucosubstance was seen in normal specimens; the secretion in these was made up of sulphated and nonsulphated acid mucosubstances, with the former predominating in the deeper half and the latter in the superficial half of the mucosa.

Benign tumours contained moderate amounts of mucosubstance, predominantly acid nonsulphated mucosubstances. Differences in the pattern of secretion were observed between the adenomatous polyp on the one hand and the villous papilloma and the papillary parts of the papillary adenoma on the other.

Carcinomas of colon and rectum contained very little secretion, with very scanty or no evidence of sulphation. The mucosa around carcinomas and in areas of non-polyploid hyperplasia in carcinoma-bearing colons showed two constant patterns, one consisting of very small amounts of secretion, with sulphated mucosubstance predominating, and the other showing normal or increased amounts of secretion almost entirely of the acid nonsulphated variety. The latter pattern extended for several centimetres from the tumour.

The possibility that sulphated mucosubstance might have a role in controlling cell division and that a decrease in its secretion might initiate a sequence of changes leading to malignancy is discussed.

The mucosubstances secreted by the human colon and rectum appear to differ from the normal, both quantitatively and qualitatively, in various diseases (Laurén, 1961; Greco, Lauro, Fabbrini, and Torsoli, 1967; Hellstrom and Fisher, 1967; Filipe, 1969; Gad, 1969; Filipe and Dawson, 1970).

This paper deals with an attempt to demonstrate the various patterns of secretion of sulphated and nonsulphated acid mucosubstances and neutral mucosubstances in benign and malignant tumours of the colon and rectum.

\section{Material and Methods}

CONTROLS

The controls consisted of 20 sections of colon and rectum from surgical material and 34 from necropsy material. The surgical specimens were from morphologically normal areas at some distance (usually

${ }^{1}$ Present address: Department of Pathology, St Tydfils Hospital, Merthyr Tydfil, Glamorgan. at least $5 \mathrm{~cm}$ ) from any lesion in colons resected for malignancy or vascular disease.

The necropsy material consisted of four specimens from an adult and 30 from infants, with no clinical or pathological evidence of colonic disease, and showing comparatively little autolytic change on routine histology.

\section{BENIGN TUMOURS}

Twelve surgically resected benign tumours were examined. Six of these were adenomatous polyps, five were papillary adenomas, and one had the structure of a villous papilloma.

\section{MALIGNANT TUMOURS}

Thirty-two specimen of rectums and colon resected for carcinoma were examined. All specimens were opened and cleaned as soon as they were received and fixed for 24 to 48 hours in $10 \%$ formalin. Sections were taken from the tumours and the adjacent mucosa and from any other mucosal lesions seen in the specimen. 
FIXATION AND PROCESSING

The tissues were fixed in $10 \%$ formalin for 24 to 48 hours, sectioned at a thickness of $5 \mu$, and stained with haematoxylin and eosin and by an Alcian blueperiodic-acid Schiff (AB-PAS) sequence (Culling, 1963), the Alcian blue staining being done at $p \mathrm{H} 1.0$ (AB 1.0-PAS) and $p \mathrm{H} 2.5$ (AB 2.5-PAS).

Some of the specimens from each group were stained by the separate Alcian blue and PAS techniques.

All photographs were taken with Kodak-Wratten fiters no. 11 green and no. 22 orange, alcianophilic material being shown up dark.

\section{Results}

Neutral mucosubstances were identified by PASpositive staining, giving a magenta to red colour with the AB 2-5-PAS technique. With this method, acid mucosubstances stained bright blue, and mixtures of acid and neutral mucosubstances took on intermediate colours.

Acid mucosubstances were defined as sulphated or nonsulphated by the AB 1.0-PAS technique, only sulphated mucosubstances staining with Alcian blue at this $p \mathrm{H}$.

\section{CONTROLS}

Controls from all levels of colon and rectum at all ages above 3 months showed a consistent pattern with sulphated mucosubstances predominating in the deeper parts of the mucosa and nonsulphated acid mucosubstances at the surface. The mucosa in between showed a mixture of sulphated and nonsulphated acid mucosubstances, with a varying proportion of the two components. Very little neutral mucosubstance was seen, many sections containing none at all. The only exception to this was seen in the ascending colon of infants below the age of 3 months, in which many glands contained a considerable amount of neutral mucosubstance.

\section{BENIGN TUMOURS}

All specimens examined showed at least moderate amounts of mucosubstance secretion.

\section{Villous papilloma}

The one specimen examined contained a very small amount of sulphated mucosubstance on the villi, the rest of the secretion being composed entirely of nonsulphated acid mucosubstance.

\section{Adenomatous polyps}

The greater part of the mucosubstance secreted was the nonsulphated acid mucosubstance with an occasional goblet cell at the base containing neutral

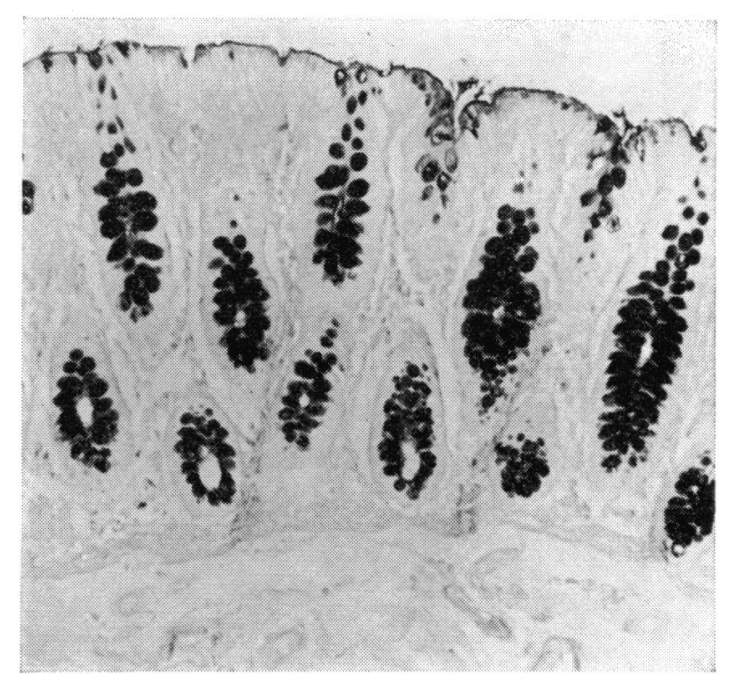

Fig. 1 Normal colon.

All the mucosubstance secreted is acid. AB 2.5-PAS $\times 120$.

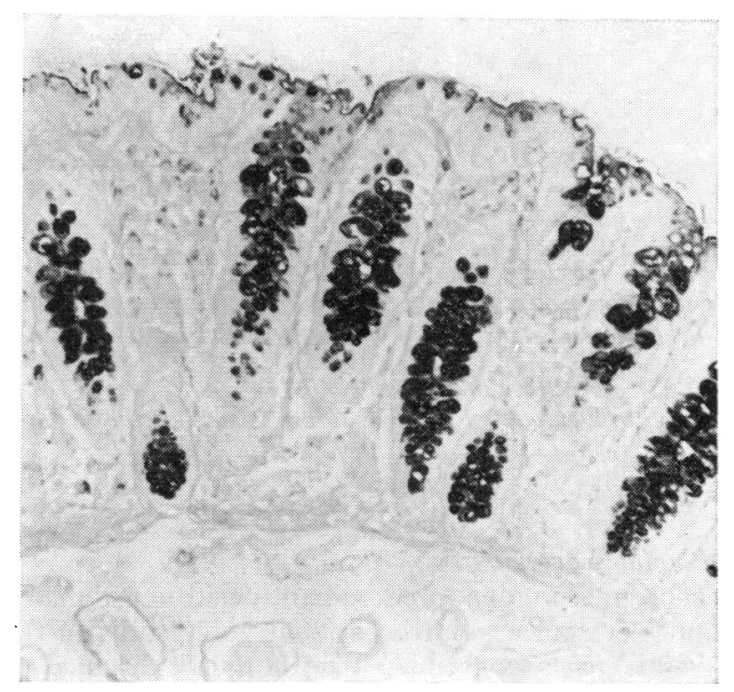

Fig. 2 Normal colon.

Most of the mucosubstance secreted is sulphated.

Nonsulphated acid mucosubstance is seen only at the surface. $A B$ 1.0-PAS $\times 120$.

mucosubstance or a mixture of neutral and acid nonsulphated mucosubstance. One specimen contained no sulphated mucosubstance apart from a thin rim along the luminal surface. The others contained scanty to small amounts of sulphated mucosubstance confined to the deeper parts of the polyps. 


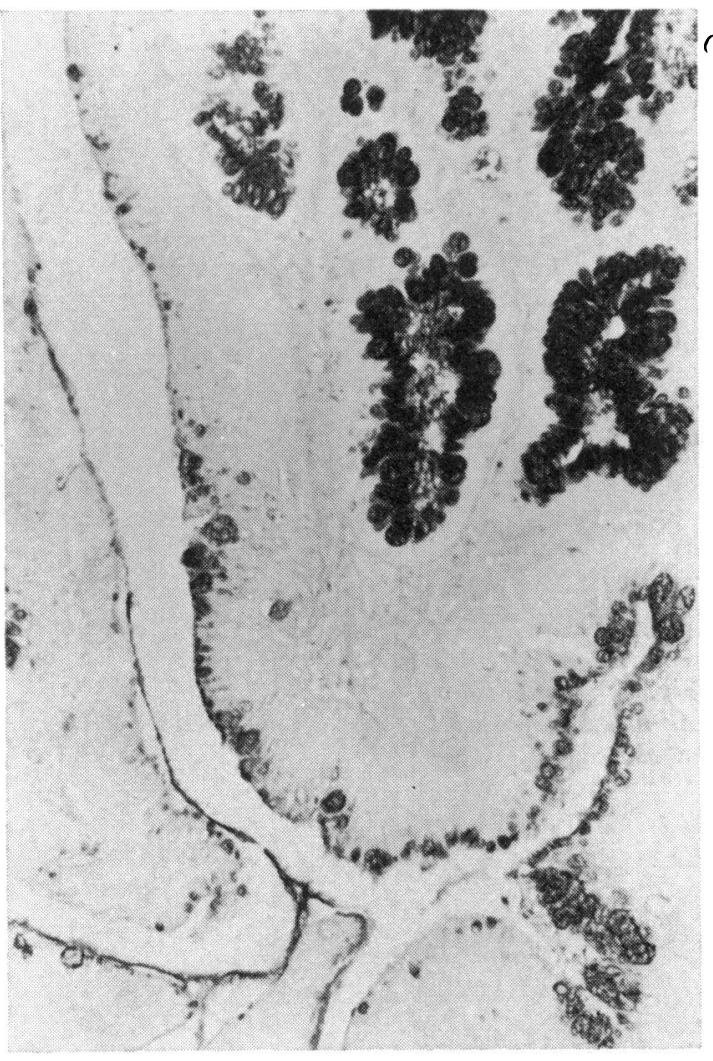

Fig. 3

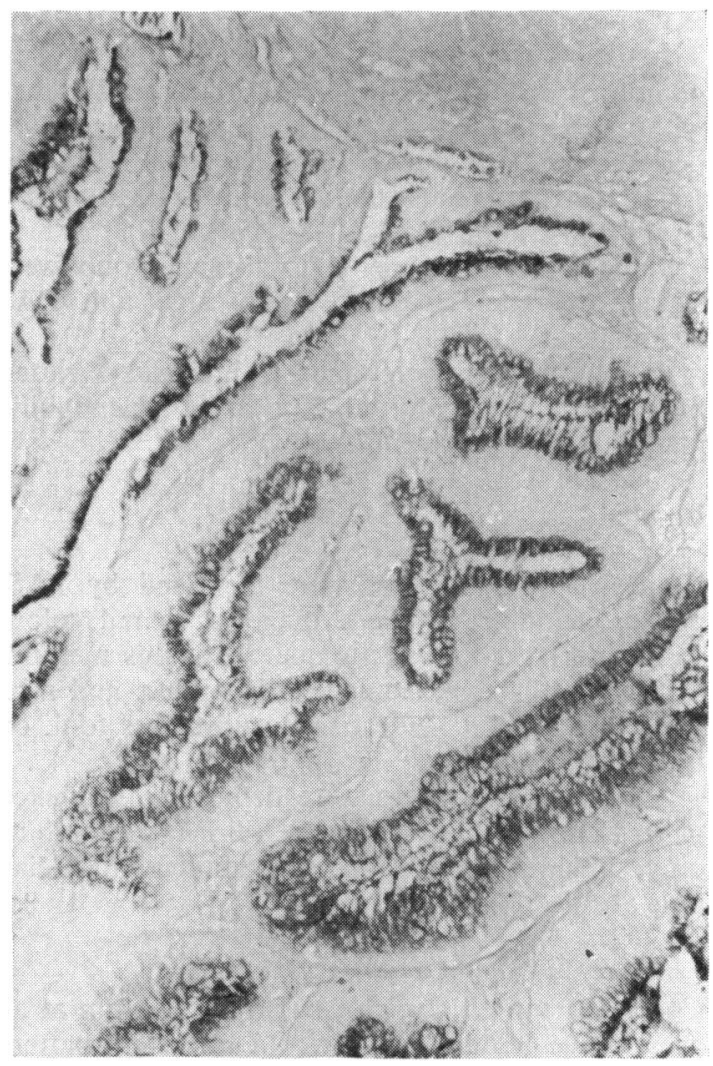

Fig. 4

Fig. 3 Papillary adenoma.

Glandular areas are lined by cells containing large amounts of acid mucosubstance. Cells at the surface contain very little mucosubstance, predominantly acid uucosubstance. AB 2-5-PAS $\times 120$.

Fig. 4 Papillary adenoma.

Very little sulphated mucosubstance is seen at the surface and in the deeper glands. AB 1.0-PAS $\times 120$.

\section{Papillary adenomas}

These too contained predominantly acid nonsulphated mucosubstance, with occasional goblet cells in the deeper parts containing neutral mucosubstance or mixtures of acid non-sulphated and neutral mucosubstances. Scanty to small amounts of sulphated mucosubstance were seen in the deeper parts in all but one case, which showed a small amount of sulphated mucosubstance on the surface of papillary processes only. The papillary processes in other cases contained less mucosubstance than the deeper parts, with very scanty to no sulphated mucosubstance.

Two types of glands could be distinguished at the depths of one papillary adenoma. One type contained small amounts of mucosubstance, none of which was of the sulphated variety. The other contained larger than normal amounts of mucosubstance, a very small part of which was sulphated, the rest being of the nonsulphated variety.

\section{MALIGNANT TUMOURS}

\section{Tumour tissue}

The tumour tissue in every case contained considerably less mucosubstance than normal, almost no secretion being observed in three cases. In general the amount of mucosubstance secreted corresponded to the extent of histological differentiation.

Of the 29 cases in which some amount of mucosubstance secretion was seen, one contained only neutral mucosubstance. In the other cases the 


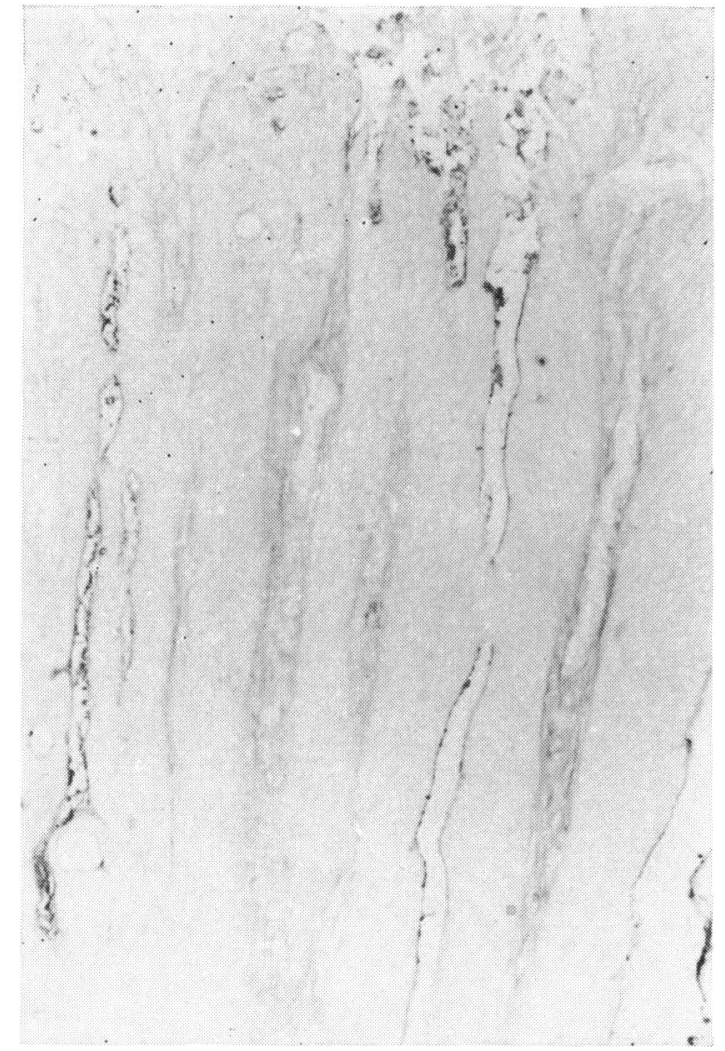

Fig. 5 Intraepithelial malignancy.

Very little mucosubstance secretion is seen. $A B 1 \cdot 0-P A S \times 120$.

secretion was made up almost entirely of acid nonsulphated mucosubstance or mixtures of acid nonsulphated and neutral mucosubstance.

No sulphated mucosubstance was observed in 12 cases. The remaining cases contained scanty sulphated mucosubstance, arranged in many cases in the form of a ring along the periphery of mucous globules.

No relationship could be established between the pattern of secretion of mucosubstance and the degree of differentiation of tumours.

\section{Mucosa adjoining tumour}

Immediately outside the tumour tissue a thin strip was seen showing very little secretory activity; a large part of the secretion in this area was of the sulphated variety. In many cases this strip was only one or two glands in thickness.

The mucosa outside this strip appeared abnormal for a varying distance, amounting to several centimetres in some cases. The glands in this area con- tained normal, or more commonly, increased amounts of secretion, almost entirely of acid nonsulphated variety, with very little or no sulphated mucosubstance and no neutral mucosubstance.

\section{Other mucosal lesions seen in specimens of bowel} bearing a carcinoma

Fourteen of the 32 specimens showed one or more small mucosal lesions apart from the carcinoma. Twenty-seven such lesions were obtained from these 14 cases. Eleven of these lesions were classified as adenomatous polyps, nine as 'non-polypoid hyperplasias $^{1}$, one as a papillary adenoma, one as a sessile area of intraepithelial malignancy, four as adenomatous polyps with areas of intraepithelial malignancy, and one as an adenocarcinoma with invasion confined to the mucosa. The term 'intraepithelial malignancy' has been used here for lesions showing marked cellular atypia and crowding with a number of mitoses, but no evidence of invasion even within the mucosa.

The lesions described as non-polypoid hyperplasias were sessile lesions, in many cases confined to the superficial part of the mucosa. They were made up of large glands, with the lining thrown up into papillary folds. The lining cells were large and in many cases had foamy or granular cytoplasm. Two types of mucosubstance secreted were seen in these. In one type normal or slightly less than normal amounts of secretion were seen, composed entirely of nonsulphated acid mucosubstance. In the second type very little secretion was seen, made up almost entirely of sulphated mucosubstance. In some cases both types of glands could be seen within the same lesion, the first type nearer the surface, while the second type occurred at the depths.

The glands in the adenomatous polyps contained varying amounts of secretion, from less to greater than normal. The mucosubstances secreted were almost entirely nonsulphated. In two cases moderate amounts of sulphated mucosubstance were seen; the total amount of secretion in both cases was much higher than normal so that the proportion of sulphated mucosubstance to total mucosubstance was very low.

The papillary adenoma contained varying amounts of secretion in different parts, composed predominantly of nonsulphated acid mucosubstance, with neutral mucosubstance in an occasional goblet cell. A small amount of sulphated mucosubstance was

\footnotetext{
'The term 'non-polypoid hyperplasia' has been used here for the lesion that has been referred to as 'metaplastic polyp 'or 'hyperplastic polyp'. I have avoided the term 'metaplastic polyp' as it does not show true metaplasia into a type of tissue normally found at some other site. I have called these lesions 'non-polypoid', reserving the term 'polyp' for lesions showing a definite stalk.
} 


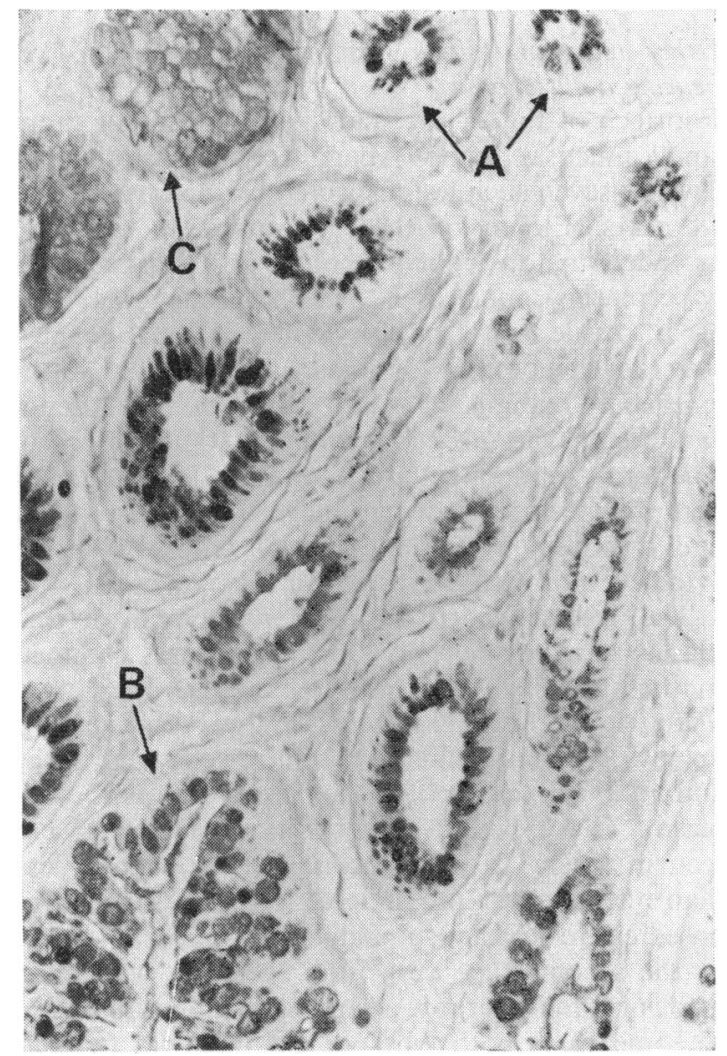

Fig. 6

Fig. 6 Non-polypoid hyperplasia.

Zone $A$ at the base consists of glands with very little secretion, most of which is sulphated. Zone B near the surface is made up of glands, with the lining thrown into papillary folds. The cells contain more secretion than zone A, almost all of it nonsulphated. (The dark staining areas in the gland marked is made up of mucin globules staining pinkish red, the dark appearance being because the staining is more solid.) Zone $C$ at the edge is made up of glands containing a large amount of secretion, all of it nonsulphated. AB $1 \cdot 0-P A S \times 120$.

Fig. 7 Edge of a carcinoma.

Arrows point to glands containing very little secretion, almost entirely sulphated. AB 1.0-PAS $\times 120$.

Fig. 8 Mucosa about a centimetre from a carcinoma. The glands are stuffed with large amounts of acid mucosubstance. AB 2.5-PAS $\times 120$.

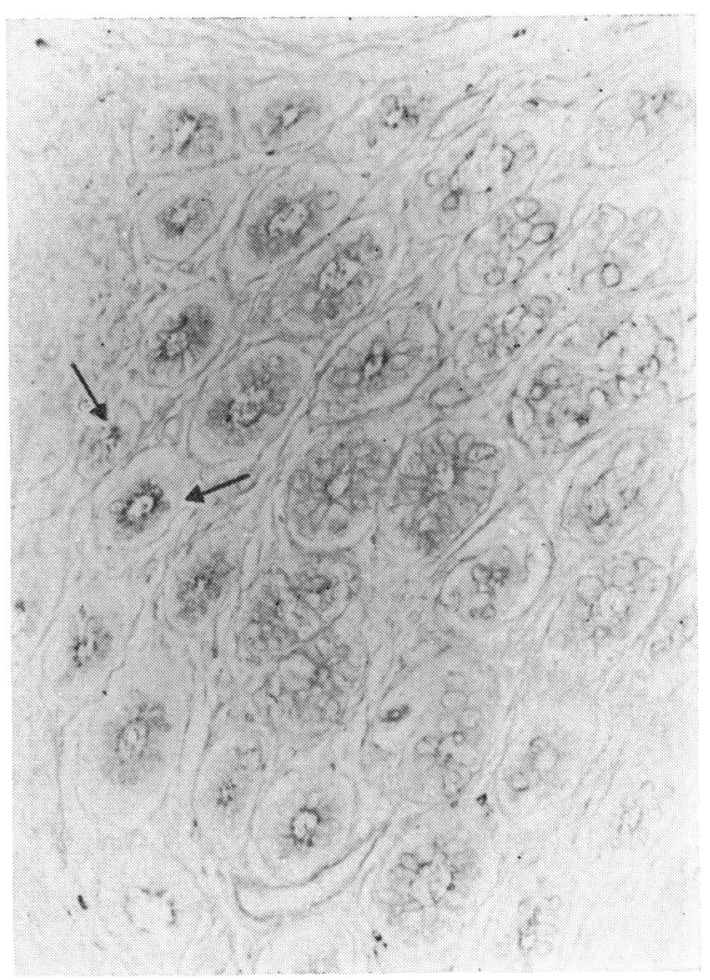

Fig. 7

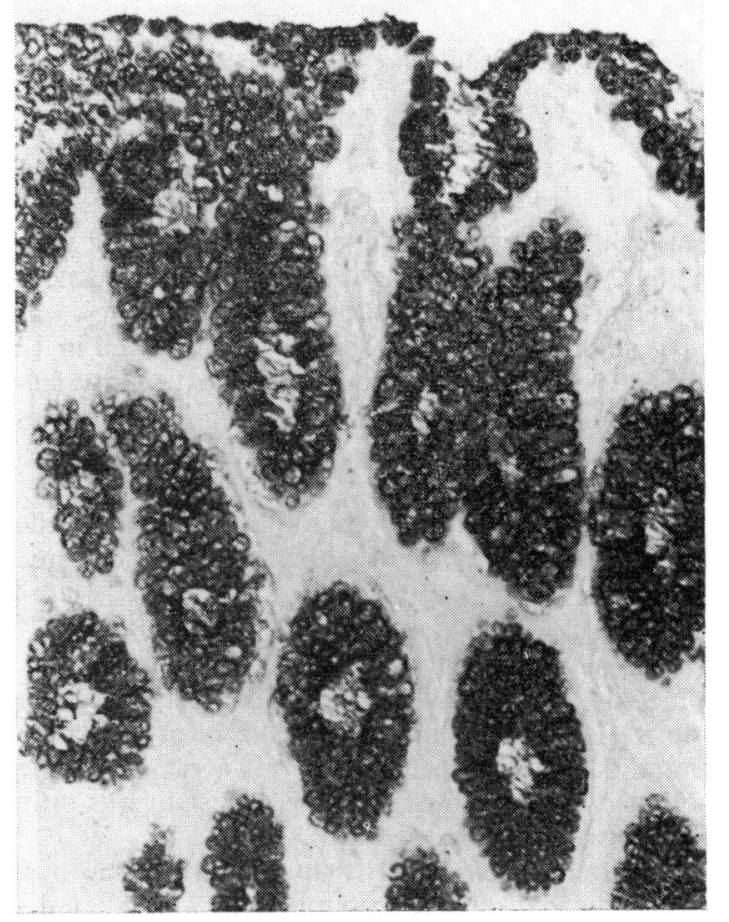

Fig. 8 
seen both on the surface of papillary processes and in the deeper parts.

The sessile area of intraepithelial malignancy contained very little secretion, made up entirely of nonsulphated acid mucosubstance. In the polyps with intraepithelial malignancy, the adenomatous areas showed the same patterns as those described above in the simple adenomatous polyps. The malignant area in three contained very little secretion, almost entirely of nonsulphated acid mucosubstance. The fourth showed more secretion-still, however, less than normal. A large part of this was sulphated and a small amount of neutral mucosubstance was also seen.

The one lesion identified as a carcinoma showed small amounts of neutral and nonsulphated acid mucosubstance, with no sulphated mucosubstance.

The patterns of secretion did not appear to be related in any way to the part of large bowel affected.

\section{Discussion}

The normal patterns of mucosubstance in the human colon and rectum have been described by various authors (Laurén, 1961; Lev and Spicer, 1965; Greco et al, 1967; Goldman and Ming, 1968; Filipe, 1969; Gad, 1969; Johansen and Kay, 1969; Filipe and Dawson, 1970). The presence of sulphated and nonsulphated acid mucosubstance is generally agreed upon, though there is some difference of opinion regarding their distribution. Thus Lev and Spicer (1965) found cells containing sulphomucin to be more numerous in the upper half of crypts. This is directly opposed to the results obtained by Filipe (1969) and Gad (1969). The results in the present series agree closely with those of the latter authors, with sulphated mucosubstance predominating in the deeper parts of the mucosa. It is interesting to note that the results obtained by Filipe with the high iron diamine technique are very similar to those obtained in the present series with the AB 1-0-PAS technique. Goldman and Ming (1968) have pointed out that sulphated mucosubstances are invariably present in the human colon but not in the small intestines. This has been verified in the present series, every specimen of colon and rectum in all age groups showing sulphated mucosubstance while not a single section of normal small bowel showed any secretion.

There is a considerable difference of opinion regarding the presence of neutral mucosubstances in the large intestines. Greco et al (1967) stated that neutral mucins could be demonstrated in all regions of the large intestine except the sigmoid colon, at the bottom and intermediate level of crypts.
This finding does not appear to have been confirmed by any other worker. Filipe (1969) reported that moderate amounts of neutral mucosubstances could be seen at the surface and in the upper crypt. Filipe and Dawson (1970), however, reported finding only small amounts of neutral mucosubstances in the upper third of crypts in the rectum. Lauren (1961) found very little PAS-reactive material in the colon except at the surface of the rectum. Lev and Spicer (1965) and Gad (1969) have found little neutral mucosubstance secreted in the large intestines. In the present series too very little neutral mucosubstance has been found at any level in the human colon and rectum. The reason for these differences in results is not clear. The results obtained by Greco et al (1967) might have been due to difference in the $p \mathrm{H}$ at which the Alcian blue staining was done.

The patterns seen in carcinomas in the present series agree very closely with those of Filipe (1969) and Gad (1969). The significant features seem to be the presence of a wider variety of mucosubstances than is seen in the normal colon and the relatively small amount of sulphomucin secretion.

Filipe (1969) has described two types of mucosubstance secreted in adenomatous polyps. In one group, little or no mucin was seen, the little that was secreted being predominantly sulphated. This type of secretion was not seen in any of the simple adenomatous polyps in the present series, but was seen in the mucosa adjacent to carcinomas.

The second type described by Filipe (1969) showed variable amounts of secretion, again predominantly sulphated. This too was not seen in the present series, the characteristic picture being the presence of variable amounts of mucin, almost entirely of the nonsulphated acid variety. A similar picture was described by Gad (1969). Only two cases in the present series resembled those described by Filipe (1969).

The papillary processes of the papillary adenomas and the villous papilloma showed an identical pattern, different from that seen in the adenomatous polyps. A striking feature about the former was its resemblance to the pattern seen at the surface of normal colons. This probably does not mean that there is a histogenetic difference between the adenomatous polyp and the villous papilloma. Greco et al (1967) have suggested that differences in the type of secretion in superficial and deep parts of crypts may represent different stages in the life cycle of the goblet cell. The differences in the patterns of secretion in the villous papilloma and the adenomatous polyp may represent a difference in the mode of maturation of cells rather than a difference in the cell of origin. 
No significant difference in mucosubstance secreted was observed between the simple adenomatous polyps and papillary adenomas and the corresponding tumours seen in cancer-bearing colons.

The patterns observed in non-polypoid hyperplasias, intraepithelial malignancies, and in the mucosa surrounding tumours showed certain striking similarities. Two patterns were constantly observed in non-polypoid hyperplasias and in the mucosa adjoining tumours. These were: (1) a zone with very little secretion, with sulphated mucosubstance predominating, and (2) a zone of excess secretion, predominantly nonsulphated acid mucosubstance. Three of the intraepithelial malignancies showed very little secretion with nonsulphated acid mucosubstance predominating. The carcinomatous tissue too showed very little or no secretion, with very little or no sulphated mucosubstance.

The mechanism of sulphation in sulphated polysaccharides is controversial, the chief point at issue being whether sulphation takes place before or after formation of the polysaccharide chain (Brimacombe and Stacey, 1964). The mucosubstance patterns described above could be explained by the assumption that sulphation was the last event in the chain. Thus if a block occurred in the sulphation mechanism this would lead to normal or increased secretion of mucosubstance, predominantly of the non-sulphated acid variety. If a block occurred in polysaccaride formation, the total amount secreted would be less, but what little was formed would be predominantly sulphated. A block occurring at more than one level would explain the formation of very small amounts of mucosubstance with very little sulphation observed in carcinomas.

One question that appears worth investigating is whether the change in mucosubstance secretion has any causal relationship to the development of carcinoma. In other words, do the changes in secretion precede the development of malignancy, or do they merely represent a disorder of cellular function as a result of malignant change? The constancy with which changes in mucosubstance secretion occur is an interesting phenomenon. It is even more significant that changes in secretion occur for quite a distance around carcinomas. The changes observed in this series in mucosa surrounding carcinomas are very similar to those described by Filipe (1969) as transitional mucosa. There is some experimental evidence to suggest that sulphated mucosubstances might inhibit tumour growth (Lippman, 1965). This raises the possibility that the sulphated mucosubstances seen in the normal colon might have a role in controlling cell division. The predominance of sulphated mucosubstance in the deeper parts of the mucosa where cell division occurs normally would support this view. A decrease in the secretion of sulphated mucosubstance would then predispose to malignancy. Such a decrease could be due to either a decrease in the total amount of mucosubstance secreted, or a specific defect in sulphation. Hellstrom and Fisher (1967) reported that the total amount of mucosubstance secreted tended to be markedly diminished in ulcerative colitis but not in Crohn's disease of the colon. This is interesting in view of the well known relationship between ulcerative colitis and colonic carcinoma. Filipe and Dawson (1970) have confirmed this finding, and added that in general the mucosubstance that remained in ulcerative colitis seemed to be rather more acid nonsulphated than sulphated. Assuming that sulphated mucosubstances control cell division, mucosa in which such change has occurred would show increase in the rate of cell division, resulting in hyperplasia; the chances of mutation occurring would also be increased, resulting in a tendency to malignant change.

The patterns of secretion seen in this series would support such a sequence. First there is a field change over a relatively wide area with secretion of normal or increased amounts of mucosubstance secretion, with little evidence of sulphation. Within this area, one or more small foci show a further change, consisting in diminution in the total amount of secretion. These two changes would explain the spectrum of patterns observed in benign neoplasms and in nonpolypoid hyperplasias. It is possible that these areas of hyperplasia are reversible and that very few of them progress to malignancy (Crocker and Veith, 1964). If they do progress to malignancy a further change in secretion occurs, with perhaps blocks at more than one level of synthesis. Bockus, Tachdjian, Ferguson, Mouhran, and Chamberlain (1961) have suggested that there are varying degrees of 'colon susceptibility' to carcinomas and polyps, and that increased susceptibility to one is often accompanied by increased susceptibility to the other. The multiplicity of mucosal lesions in colons bearing a carcinoma has been pointed out by several authors (Helwig, 1947; DeMuth, Cherney, and Fitts, 1952; Moertel, Bargen, and Dockerty, 1958; Bockus et al, 1961; Crocker and Veith, 1964; Wood, 1967). These features could be explained by a series of events such as those described above.

One way of ascertaining if such a sequence occurs would be to study conditions known to predispose to malignancy, such as ulcerative colitis. Morson and Pang (1967) have described changes suggestive of precancer or carcinoma in situ in cases of ulcerative colitis. It would be of interest to study the patterns of mucosubstance secreted in these foci, 
and to see if field changes of the type suggested occur in these cases.

My thanks are due to Professor B. E. Tomlinson for permission to use material from the files of the Newcastle General Hospital and for the constant encouragement I have received from him; to Dr T. Bird, consultant pathologist, Newcastle General Hospital, for lending me his collection of necropsy material of intestines of infants and children, and to the technical personnel of the Pathology Department, Newcastle General Hospital, particularly Mr M. J. Stones, Mr D. S. Smith, and Miss D. Kitchener.

\section{References}

Bockus, H. L., Tachdjian, V., Ferguson, L. K., Mouhran, Y., and Chamberlain, C. (1961). Adenomatous polyp of colon and rectum: its relation to carcinoma. Gastroenterology, 41, 225-232.

Brimacombe, J. S., and Stacey, M. (1964). Mucopolysaccharides in disease. Advance. in clin. Chem., 7, 199-234.

Crocker, D. W., and Veith, F. J. (1964). Focal primary mucosal hyper-plasia of the colon. Ann. Surg., 160, 215-225.

Culling, C. F. A. (1963). Handbook of Histological Techniques. 2nd ed., p. 233. Butterworth, London.

DeMuth, W. E. Jr., Cherney, P. J., and Fitts, W. T. Jr. (1952). Adenomatous polyps of the colon and rectum. Surg. Gynec. Obstet., 94, 195-199.
Filipe, M. I. (1969). Value of histochemical reactions for mucosubstances in the diagnosis of certain pathological conditions of the colon and rectum. Gut, 10, 577-586.

Filipe, M. I., and Dawson, I. (1970). The diagnostic value of mucosubstances in rectal biopsies from patients with ulcerative colitis and Crohn's disease. Gut, 11, 229-234.

Gad, A. (1969). A histochemical study of human alimentary tract mucosubstances in health and disease. I Normal and tumours. II Inflammatory conditions. Brit. J. Cancer, 23, 52-68.

Goldman, H., and Ming,S.C. (1968). Mucins in normal and neoplastic gastrointestinal epithelium: histochemical distribution. Arch. Path., 85, 580-586.

Greco, V., Lauro, G., Fabbrini, A., and Torsoli, A. (1967). Histochemistry of the colonic epithelial mucins in normal subjects and in patients with ulcerative colitis. Gut, 8, 491-496.

Hellstrom, H. R., and Fisher, E. R. (1967). Estimation of mucosal mucin as an aid in the differentiation of Crohn's disease of the colon and chronic ulcerative colitis. Amer. J. clin. Path., 48, 259-268.

Helwig, E. B. (1947). The evolution of adenomas of the large intestine and their relation to carcinoma. Surg. Gynec. Obstet., 84, 36-49.

Johansen, P. G., and Kay, R. (1969). Histochemistry of rectal mucus in cystic fibrosis of the pancreas. J. Path., 99, 299-306.

Laurén, P. (1961). The cell structure and secretion in intestinal cancer. Acta path. microbiol. scand., Suppl. 152.

Lev, R., and Spicer, S. S. (1965). A histochemical comparison of human epithelial mucins in normal and hypersecretory states including pancreatic cystic fibrosis. Amer. J. Path., 46, 23-47.

Lippman, M. (1965). A proposed role for mucopolysaccharides in the initiation and control of cell division. Trans. N.Y. Acad. Sci., 27, 342-360.

Moertel, C. G., Bargen, J. A., and Dockerty, M. B. (1958). Multiple carcinomas of the large intestine. Gastroenterology, 34, 85-98.

Morson, B. C. , and Pang, L. S. C. (1967). Rectal biopsy as an aid to cancer control in ulcerative colitis. Gut, 8, 423-434

Wood, D. A. (1967). Tumours of the Intestines, Atlas of Tumour Pathology, Sect. 6, Fasc. 22. Armed Forces Institute of Pathology, Washington, D.C. 